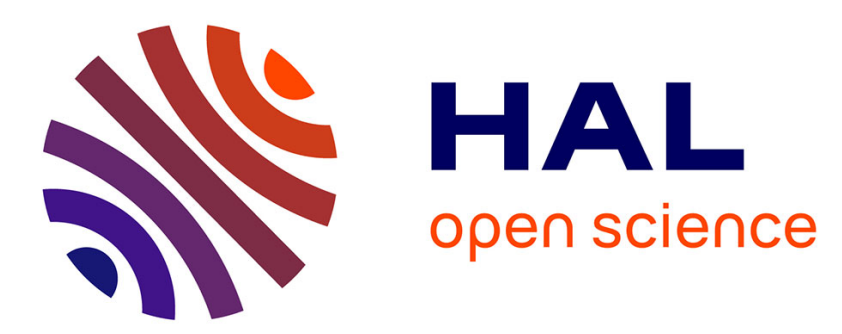

\title{
Le sel dans le nord-est de la France et le sud-ouest de l'Allemagne: une approche des structures de production et d'échange aux Âges du Fer
}

Clara Millot-Richard, Olivier Weller

\section{To cite this version:}

Clara Millot-Richard, Olivier Weller. Le sel dans le nord-est de la France et le sud-ouest de l'Allemagne: une approche des structures de production et d'échange aux Âges du Fer. Sel et société. Tome 2: Santé, croyances et économie, 2020. hal-03035746

\author{
HAL Id: hal-03035746 \\ https://hal.science/hal-03035746
}

Submitted on 2 Dec 2020

HAL is a multi-disciplinary open access archive for the deposit and dissemination of scientific research documents, whether they are published or not. The documents may come from teaching and research institutions in France or abroad, or from public or private research centers.
L'archive ouverte pluridisciplinaire HAL, est destinée au dépôt et à la diffusion de documents scientifiques de niveau recherche, publiés ou non, émanant des établissements d'enseignement et de recherche français ou étrangers, des laboratoires publics ou privés. 
Le sel dans le nord-est de la France et le sud-ouest de l'Allemagne : une approche des structures de production et d'échange aux Âges du Fer.

\title{
Clara Millot-Richard et Olivier Weller ${ }^{1}$
}

\section{Résumé}

Le sel, matière minérale à la fois essentielle et invisible pour l'archéologie, est utile à l'alimentation humaine et animale, à la conservation de viande ou de fromage, intègre d'autres artisanats, mais également a pu servir, à certains moments précis, de réserve de valeur, voire de valeur étalon d'échange. Il s'agira d'appréhender les productions de sel aux Âges du fer à l'échelle régionale et macro-régionale, ici le nord-est de la France et le sudouest de l'Allemagne, avérées ou supposées, en mettant en évidence les agents et les échelles de production en fonction des contextes. La confrontation avec d'autres circuits de production, comme celui du fer, viendra enrichir les réflexions et ouvrir de nouvelles pistes de recherche à explorer.

\begin{abstract}
Salt is a raw material both crucial and invisible in archaeology. It is used in human and animal food supply, in meat and dairy preservation, in other crafts but might also have had a role of storage of values and standard of value. This paper is dealing with ways to apprehend salt productions (proven and suspected) during the Iron Age at a regional and macro-regional scale, more precisely north-eastern France and south-western Germany by highlighting producing agents in their different contexts. The confrontation with other production network, such as iron's, will extend reflexions and open new lines of research.
\end{abstract}

\section{Introduction}

Il ne fait aucun doute que le sel est une matière première cruciale dans de nombreux systèmes économiques, et ce depuis l'apparition des premières économies de production en Europe dès le début du Néolithique ${ }^{2}$. C'est d'autant plus vrai pour les économies protohistoriques, où le sel sert à la confection de salaisons, à l'alimentation animale, à l'artisanat, mais également probablement comme réserve de valeur. Débutée dès l'Âge du Bronze moyen, et poursuivie lors des Âges du Fer européens, une exploitation véritablement intensive des ressources en sel émerge et va être à l'origine de dynamiques économiques et de réseaux d'échanges plus ou moins développés, la Tène finale marquant un véritable boom de la production à l'échelle européenne $^{3}$ (Fig. 1). Matière première si importante, mais invisible et dont les agents producteurs et surtout consommateurs sont si difficiles à cerner.

\footnotetext{
${ }^{1}$ UMR 8215-Trajectoires, Université Paris 1 Panthéon-Sorbonne / CNRS ; claramiri@wanadoo.fr ; olivier.weller@cnrs.fr

${ }^{2}$ WELLER 2015.

${ }^{3}$ FRIES-KNOBLACH 2001 ; SAILE 2002
} 
Cet article se propose d'aborder les systèmes de production et d'échange du sel durant les Âges du Fer (Hallstatt et La Tène) dans le nord-est de la France et le sud-ouest de l'Allemagne. Les deux sous périodes des Âges du Fer européens voient avec le Hallstatt (620480 av. J.-C.) l'apparition du phénomène des «résidences princières », une certaine concentration des savoir-faire et des richesses ${ }^{4}$; puis avec La Tène (480-26 av. J.-C.) l'apparition progressive des cités gauloises et de la monnaie métallique frappée ${ }^{5}$.

Dans ces deux régions, les dynamiques économiques liées à l'exploitation des matières premières minérales sont particulièrement riches d'information, en raison de la concentration d'agents producteurs et consommateurs. Les sites de production de sel, aussi bien dans la vallée de la Seille (Moselle) qu'à Schwäbisch Hall (Bade-Wurtemberg), comptent parmi les mieux connus en contexte continental (Fig. 2). Citons également les salines voisines de Franche-Comté ${ }^{6}$ et celle de Bad Nauheim ${ }^{7}$ en Hesse qui ont également fonctionné durant ces périodes. Si la matière première est abondante, mais plus diluée, sur les littoraux (l'exploitation du sel est attestée sur presque toutes les façades maritimes), elle est plus rare en contexte continental. En dehors des mines proprement dites (Hallstatt et Hallein-Dürnberg en Autriche), c'est sous la forme de résurgences d'eau salée plus ou moins concentrée que l'on retrouve le $\mathrm{sel}^{8}$. En plus de celles déjà citées, on connaît d'autres sources minérales chlorurées sodiques dans les Pyrénées, les Alpes, le Massif Central ou encore dans le centre de l'Allemagne. Cette relative rareté et hétérogénéité dans la distribution des ressources conféraient très probablement un surcroît de valeur au sel tout particulièrement dans les bassins sédimentaires comme le Bassin parisien. Ces dynamiques ouvrent la voie à des questionnements sur les échelles et le contrôle des productions, la notion de surplus et sur la valeur de ce sel protohistorique. Le sel, ou plutôt devrions dire les sels, pouvaient servir à de multiples usages comme le soulignent le recensement exhaustif des textes antiques ${ }^{9}$ : l'alimentation humaine bien entendu, la conservation alimentaire, la nourriture des animaux, la fromagerie, la tannerie, la teinturerie, mais aussi la pharmacopée, la parfumerie, la métallurgie, l'architecture, ou encore dans divers rituels pour son fort pouvoir symbolique.

Toutefois, les marqueurs archéologiques dont nous disposons pour percevoir les utilisations et la distribution du sel protohistorique sont minces et, quasi sans source textuelle exploitable, ils sont les seuls éléments sur lesquels compter. Ces marqueurs archéologiques sont constitués de fragments de briquetage (cf. infra), c'est-à-dire des fragments d'éléments d'argile plus ou moins cuite entrant dans la fabrication non pas uniquement de sel, mais de pains de sel (moule céramique, éléments de supports et de calage, fragment de fourneaux...). Ces éléments, souvent très fragmentés et de facture grossière, n'ont pas été toujours reconnu dans le cadre des fouilles anciennes, parfois aussi de fouilles récentes, ce qui biaise encore la donnée dont nous disposons.

\section{La production de sel}

\footnotetext{
${ }^{4}$ BuChSENSCHUTZ 2015, p. 108-153.

${ }^{5}$ FICHTL 2004 ; BUCHSENSCHUTZ 2015, p.295-315.

${ }^{6}$ PETREQUin \& WELLER 2008.

${ }^{7}$ KULL 2003.

${ }^{8}$ WeLler 2015.

${ }^{9}$ MOINIER \& WELlER 2015.
} 
Pour les Âges du Fer européens, la production de sel à partir d'eau salée (eau de mer, source salée...) est dite "ignigène » c'est-à-dire sous l'effet d'une chaleur artificielle. Même si les principales techniques du sel sont aujourd'hui bien cernées ${ }^{10}$, la chaîne opératoire de production protohistorique est complexe et il existe encore de nombreuses incertitudes dans son déroulement précis ${ }^{11}$. Pour résumer l'exploitation qui nous concerne ici, celle de sources minérales chlorurées sodiques, il s'agit d'abord de capter puis d'extraire, voire stocker, l'eau salée, sans doute au moyen de puits en bois comme on les connaît encore aujourd'hui dans les Carpates orientales $^{12}$. La fabrication des éléments de briquetage se fait à partir d'argile locale : les cuvettes ou baquets à sel étanches vont permettre, sous l'action du feu, de concentrer l'eau salée pour obtenir une saumure à saturation qui sera encore pousser jusqu'à l'obtention d'une pâte de sel humide ; les moules à sel de conditionner cette pâte en pains de sel de forme et poids normalisés. Les moules sont en théorie brisés une fois le pain de sel sec, afin de le récupérer ${ }^{13}$. Ce processus exige une grande quantité d'argile et de bois pour chauffer la saumure et cuire le sel, ce qui peut avoir des conséquences importantes pour les écosystèmes passés $^{14}$.

\subsection{La Seille : plusieurs centres de production}

Un des centres de production de sel connu pour les Âges du Fer européens est la vallée de la Seille (Moselle). La Seille est un affluent de la Moselle et permet de rejoindre Metz d'un côté. D'un autre côté, on peut aisément rejoindre le plateau de la Haye, ou se diriger vers les Vosges ou la vallée du Rhin. La Seille est une vallée dont le contexte géologique est favorable à la résurgence de sources à salinité très élevée. L'eau de ruissellement s'infiltre dans le soussol, riche en roches et argiles salifères, et constitue ensuite des poches d'eau salée fortement concentrée qui sourdent en plusieurs endroits de la vallée ${ }^{15}$.

Les briquetages de la Seille ont été découverts par l'ingénieur F. Le Royer de La Sauvagère ${ }^{16}$ lorsqu'il fait construire à Marsal les fortifications de la citadelle Vauban à la fin du règne de Louis XIV. A partir de ce moment, les découvertes et les recherches des érudits ou conservateurs du patrimoine s'enchainent et amènent progressivement à une compréhension de la chaîne opératoire de production du sel. ${ }^{17}$

Les ateliers d'exploitation du sel sont repérés, dans les méandres de la Seille, par la présence «d'îlots » de briquetage, c'est-à-dire des accumulations de fragments de déchets de terre cuite, des déchets de production qui peuvent être amassés sur plusieurs mètres de hauteur (entre 10 et $12 \mathrm{~m}$ pour les plus importants), et plusieurs centaines de mètres de surface. Le volume total de ces déchets est estimé entre 3 et 5 millions de $\mathrm{m}^{3}$. Quelques-uns des plus importants sont Marsal «Fort d'Orléans » et « Le Bourg », Moyenvic « Les Crôleurs ${ }^{18} »$, Vicsur-Seille «Le Châtry» et Salonnes «Burthécourt ${ }^{19}$. On compte également plusieurs

\footnotetext{
${ }^{10}$ GOULETQUER et al. 1994.

${ }^{11}$ DAIRE 2003 p. 20-25.

${ }_{13}^{12}$ AleXianu et al. 2008.

${ }^{13}$ OLIVIER 2012, p. 31-44 ; OLIVIER 2010, p.127-160 ; OLIVIER 2000, p.143-173.

${ }^{14}$ RIDDIFORD et al. 2016.

${ }^{15}$ BEAUPRE 1901 ; KEUNE 1901

${ }^{16}$ LE ROYER DE LA SAUVAGERE 1740.

${ }^{17}$ BERTAUX 1981.

${ }^{18}$ LAFITTE 2002, 2006-2010.

${ }^{19}$ OLIVIER 2006, p. 12.
} 
nécropoles tumulaires dans la vallée dont la majorité ne sont pas fouillées mais sont datées par prospection pédestre du Hallstatt $\mathrm{C}$ et $\mathrm{D}$, soit synchrone de l'apparition des premiers briquetages dans la vallée. Le projet «Briquetage de la Seille » mené par L. Olivier a permis de mener des prospections et des fouilles régulières dans la vallée depuis 2001 afin de comprendre davantage l'extension spatiale et l'organisation interne des ateliers de production $^{20}$. Quelle que soit la chronologie considérée, les habitats des Âges du Fer ne sont pas connus sur la vallée, mais les recherches géoarchéologiques en particulier apportent de nombreuses informations sur l'environnement des sauniers (et l'impact de leur exploitation du sel sur le milieu $)^{21}$.

L'exploitation du sel dans la vallée de la Seille s'établit sur la longue durée ${ }^{22}$ (Fig. 3). Les éléments de briquetages les plus anciens remontent au Hallstatt C1-C2 (soit env. 750-620 av. J.-C.) et présentent la forme de bassines à large ouverture et de formes hautes. Lors du Hallstatt D1, elles conservent la même forme mais leurs proportions diminuent légèrement tant en largeur qu'en hauteur. Un changement net apparait au Hallstatt D2-D3, où les moules à sel changent de forme. Il s'agit de godets plus hauts que larges, beaucoup plus effilés. Les formes de moules à sel utilisées lors de La Tène A-B ne sont pas connues parce que les zones d'atelier de cette époque ne sont pas reconnues sur la vallée. Il est probable que ces «îlots » du début de la période laténienne soient situés sous les îlots plus tardifs de La Tène C- $\mathrm{D}^{23}$. Les formes de moules à sel connues pour cette dernière période n'ont pas changé depuis le Hallstatt D3, malgré le hiatus de La Tène A-B. Il s'agit toujours de moules fuselés, légèrement plus étroits que les exemples hallstattiens. On note un arrêt de l'exploitation et un abandon des structures de production à la fin de la période laténienne et au début de la période romaine.

Les raisons de ce changement de forme entre le Hallstatt $\mathrm{C}$ et $\mathrm{D}$ ne sont pas bien comprises à l'heure actuelle; on suppose que la forme plus étroite des moules à sel aurait pu permettre de réduire le temps de chauffe de la saumure et éventuellement de limiter la perte de saumure lors de cette phase de chauffe ${ }^{24}$. Quoiqu'il en soit, l'exploitation des ressources salées de la vallée s'étend sur presque sept siècles, soit une quasi continuité d'occupation de la vallée si l'on excepte le hiatus apparent de la Tène A-B.

En plus de sa longue durée, l'exploitation du sel dans la vallée de la Seille s'organise d'une manière qualifiée de «proto-industrielle ». L'étendue des ateliers, le volume des déchets et le regroupement des structures de chauffe de la saumure, des structures de concentration ou de traitement de la saumure donnent un aspect intensif à la production. Il s'agit d'une production organisée et spécialisée (cf. infra) qui a très probablement modifié durablement le paysage et l'environnement de la vallée ${ }^{25}$.

Toutes ces structures exigent une main d'œuvre importante, soit plusieurs centaines de personnes d'une même génération, auxquelles il faudrait ajouter les agents producteurs de nourriture (agriculteurs, éleveurs), et les autres artisans spécialisés comme des charpentiers,

\footnotetext{
${ }^{20}$ OLIVIER 2001, p.143-171.

${ }^{21}$ JUSSERET et al., 2015, p.515-538.

${ }^{22}$ OLIVIER 2012, p. 36.

${ }^{23}$ OLIVIER 2010, p. 127-160.

${ }^{24}$ DAIRE, 2003.

${ }^{25}$ RIDDIFORT et al., 2016.
} 
des métallurgistes etc. Les quelques estimations démographiques proposées pour l'époque du Hallstatt envisagent quelques cinq mille personnes par génération dans la vallée de la Seille, ce qui représente un bassin de population très important pour l'époque ${ }^{26}$.

\subsection{Un autre site de production : Schwäbisch Hall (Wurtemberg)}

Dans la zone d'étude prise en compte, il existe un seul site de production de sel attesté. Il s'agit du site de Schwäbisch Hall situé dans le Bade-Wurtemberg ${ }^{27}$. Cette ville est située dans la plaine du Hohenhole, sur le cours du Kocher, affluent du Neckar.

Ce site fouillé dans les années 1939-1940, à l'occasion de travaux de canalisations sur une petite surface, a livré une quantité importante de céramique et un nombre très important de fragments de briquetage (environ 30000 fragments, Fig. 4), ainsi que plusieurs structures en bois dont un puits, un plancher, trois réservoirs aménagés dans des troncs creusés (Fig. 5), un conduit en bois et deux bassins tapissés d'argile ${ }^{28}$. La fouille n'a pu être étendue au-delà du sondage créé pour ces travaux, et on peut donc supposer que ce site a un potentiel archéologique beaucoup plus important. De plus, toute une partie du mobilier a été perdu au cours du second conflit mondial, ce qui a interdit une étude complète du mobilier.

C'est le seul site de production connu à l'heure actuelle pour le Bade-Wurtemberg et, contrairement à la Lorraine, nous disposons de cartes de répartition de briquetages sur des sites a priori autres que des sites de production. Avant même d'évoquer la typologie de ces briquetages, on peut remarquer que ces briquetages sont essentiellement présents dans le nord-est du Bade-Wurtemberg, dans la vallée du Neckar, bien qu'on en compte quelques occurrences dans le Jura souabe. On suppose donc que ces briquetages proviennent tous du site de Schwäbisch Hall ${ }^{29}$.

Comme dans la vallée de la Seille, on retrouve une occupation sur la longue durée dont témoigne la typologie des briquetages (Fig. 6). Pour le Hallstatt D3-LTA, on retrouve des briquetages dits «U $\mathrm{U}$ » et «Ugross » en raison de leur forme ; les formes évoluent ensuite vers celles dites en « 7 », qui apparaissent probablement au cours de La Tène B, mais les sites de cette période étant rares, la transition est difficile à saisir. Ils perdurent au cours de La Tène $\mathrm{C}$ et $\mathrm{D}$, mais durant ces phases de La Tène moyenne et finale des formes en « $\mathrm{P} »$ plus arrondies et ayant une contenance plus élevée que les autres formes $\left(1700 \mathrm{~cm}^{3}\right)$ se développent également.

A partir des données disponibles, le site de Schwäbisch Hall apparaît comme une production importante à l'échelle régionale, mais qui ne semble pas avoir l'envergure d'une région productrice comme la Seille. En effet, aucun matériel «prestigieux » ou d'origine exogène n'a été découvert dans les quelques sondages ouverts : pas d'ambre balte, pas de corail, pas de lignite ni de céramique décorée au graphite.

\footnotetext{
${ }^{26}$ OLIVIER 2000, p. 143-173.

${ }^{27}$ HEES, 2002.

${ }^{28}$ HOMMEL, 1940, p. 129-144 ; KOST, 1940, p.39-111 ; VEECK 1940.

${ }^{29}$ HeEs 2002, p. 209-217.
} 
Toutefois, il ne faut pas négliger la présence de nombreuses sources salées dans la région septentrionale de Heilbronn ${ }^{30}$ qui auraient potentiellement pu être exploitées aux époques protohistoriques $^{31}$. Etant donné l'importante quantité de briquetages retrouvés sur de très nombreux sites de cette micro-région de la vallée du Neckar (Fig. 7), datés autant du Hallstatt final que de La Tène, on peut se demander s'il n'existerait pas d'autres sites de productions qui n'auraient pas pu être identifiés notamment à cause de l'urbanisation importante de cette zone.

La saline de Bad Nauheim dans la Wetterau (Hesse) est également un site de production très important (mais situé en dehors de notre zone d'étude) qui a fonctionné de La Tène B2 jusqu'à la période romaine. Des fouilles ont été menées en 1953 et plus récemment entre 2001-2004, et elles ont révélé des bassins réservoirs pour la saumure en bois, des structures de bassins avec pavés, des vases de chauffe etc ${ }^{32}$. Dans ce cas la production du sel, est couplée avec de la production d'objets en fer, de bronze et de certains matériaux précieux comme l'ambre. La nature des relations concurrentielles entre Bad Nauheim et Schwäbisch Hall reste encore à définir précisément.

\section{Questionnements économiques}

\subsection{Les exploitants du sel}

Au sujet de l'organisation spécifique de la production de sel, il est probable que les différentes étapes de la production ne soient pas effectuées par les mêmes agents. Il n'existe que très peu d'éléments qui nous renseignent sur la main d'œuvre de ces exploitations. Les éléments de parure en lignite en cours de taille trouvés dans les couches Hallstatt C2-D1 de certains ateliers de la vallée de la Seille sembleraient indiquer que les personnes travaillant dans ces ateliers étaient capables d'importer et de posséder cette matière précieuse ${ }^{33}$. Mais à l'inverse, il n'existe aucune preuve archéologique qui permettrait d'affirmer que les travailleurs du sel de la Seille n'étaient pas des esclaves. En effet, le travail du sel est difficile, la saumure est une matière particulièrement corrosive pour la peau, la chaleur et les fumées devaient très présentes et on pourrait aisément comprendre qu'une partie de la production ait été déléguée à des esclaves. On peut également évoquer une division sexuelle du travail. Plusieurs exemples ethnographiques $^{34}$ montrent l'importance de la part des femmes dans le travail du sel, notamment dans le traitement et la concentration de la saumure ${ }^{35}$. On en tient pour preuve la présence de bracelets en roche noire (ou lignite) retrouvés dans la zone d'ateliers de « La Digue », qui sont des éléments de parure retrouvés dans les riches sépultures féminines de l'époque, car la roche noire est un matériau précieux qui vient de loin. On peut donc tout à fait supposer la présence de femmes, et donc de femmes probablement aristocrates, sur les ateliers de traitement de la saumure dans la vallée de la Seille.

\footnotetext{
${ }^{30}$ CARLE 1965

${ }^{31}$ HEES 2012, p. 277-286

${ }^{32}$ KULL 2003.

${ }^{33}$ OLIVIER, 2010, p. 127-160.

${ }^{34}$ Weller 2006 ; OLIVIER, 2010, p. 127-160.

${ }^{35}$ OLIVIER, 2013, p. 175.
} 
De l'organisation de ces productions, on peut déduire que le sel façonné est essentiellement destiné à du surplus et voué à circuler. Même si l'exploitation de Schwäbisch Hall semble moins intensive ou moins étendue que celle du saulnois lorrain, il est clair que dans les deux cas, le sel est destiné à une consommation supra-régionale et non à une consommation locale par les agents producteurs eux-mêmes. On peut également s'interroger sur la gestion et la place que tenait la constitution de ces surplus au sein de ces communautés de sauniers.

\subsection{Les échelles de la distribution}

Il va de soi que la question de l'échelle de ces exploitations est un élément fondamental pour comprendre les implications économiques de l'exploitation protohistorique du sel. Il est évident que la production de sel du saulnois lorrain, mais également celle de Schwäbisch Hall dans le Wurtemberg dépassent le cadre d'une production locale. Il s'agit dans les deux cas de productions l'une régionale, l'autre supra régionale, qu'il convient de distinguer. Les structures de production de la Seille montrent qu'elles sont reliées à un réseau d'échanges à longue distance, comme l'illustrent par exemple la présence dans la vallée de bracelets incrustés de corail ou celles de petites perles en ambre ${ }^{36}$. Des analyses isotopiques sur les dents de suidés retrouvés sur les sites d'ateliers de la Seille ont montré qu'une partie du cheptel venait des Vosges, et il est probable qu'une partie du sel produit devait être exporté vers ces régions ${ }^{37}$. Si les trop rares découvertes de briquetages à l'extérieur de la vallée n'ont pas permis de préciser les débouchés commerciaux, les fouilles préventives récentes de la LGV-Est en Alsace sur le site d'Entzheim Geispolsheim ont mis au jour des fragments de barres $^{38}{ }^{39}$. Ces barres de briquetage proviennent de la vallée de la Seille et datent de La Tène A. La distance parcourue entre le site de production et le site de consommation est $120 \mathrm{~km}$ à vol d'oiseau. Pour le Bade-Wurtemberg, les données que nous possédons sur la répartition des briquetages montrent que les pains de sel étaient distribués à au moins une centaine de kilomètres du site de production pour la période du Hallstatt D3- La Tène A et à environ 70 $\mathrm{km}$ pour la période La Tène C-D ${ }^{40}$. Au premier coup d'œil, il semblerait que le site de Schwäbisch Hall distribue à moins grande échelle que la vallée de la Seille, toutefois quelques briquetages badois ont été retrouvés sur des sites localisés au-delà du Jura souabe, voire même en Bavière ${ }^{41}$. De plus, il faut distinguer briquetage et consommation du sel car, si le pain de sel a été débarrassé de son moule en terre cuite à un endroit $\mathrm{X}$, ce même bloc de sel, complet ou divisé, a pu être consommé plus loin encore.

2.3 «Proto-industrialisation» et normalisation des produits: repenser les industries anciennes.

La division du travail et la segmentation de la chaîne opératoire de production s'accompagnent aussi d'une forte normalisation des productions. En effet, on constate que les pains de sels d'une région productrice pour une période donnée ont une forme et une taille et sans doute un poids relativement standardisé. Ainsi, à époque équivalente, les productions du saulnois lorrain avaient un aspect, une forme et un poids de sel différent et différenciable de

\footnotetext{
${ }^{36}$ OLIVIER, 2007, p. 5-14.

37 OLIVIER, 2000.

${ }^{38}$ FLEISCHER, LANDOLT, 2012, p.193-207 ; LANDOLT ET AL, 2008.

${ }^{39}$ LANDOLT et al, 2008.

${ }^{40}$ HeEs, 2002, p. 209-2017.

${ }^{41}$ HeEs, SCHARFF, 2003, p. 133-138.
} 
celles de leurs voisins de Franche-Comté, du Bade-Wurtemberg ou de Hesse en provenant du site de Bad Nauheim ${ }^{42}$. Ces produits étaient donc reconnaissables par les populations de l'époque. Et le sel gemme provenant de la mine de Hallstatt était lui aussi aisément identifiable, les «lingots » de sel étaient présentés sous forme de demi cœur, probablement pour en faciliter le transport et l'échange ${ }^{43}$.

Tous ces éléments nous poussent à discuter la notion «d'industrie » du sel pour les périodes protohistoriques, ainsi que de «proto-industrie» ou «d'artisanat». Selon les définitions du terme d'industrie, il s'agit de «l'ensemble des activités économiques (caractérisées par la mécanisation, la centralisation des moyens de production et la concentration de la propriété des moyens de production), ayant pour objet l'exploitation des richesses minérales ainsi que la production de produits fabriqués à partir de matières premières ou de matières ayant déjà subi une ou plusieurs transformations. ${ }^{44}$ » Nous ne disposons d'aucun moyen pour construire des hypothèses sur la propriété des moyens de production. Bien sûr, ces définitions sur l'industrie ont été pensées pour décrire la Révolution Industrielle occidentale et ne sont donc pas véritablement adaptées à nos contextes anciens et au caractère lacunaire de la donnée. Mais, compte tenu de la documentation amassée depuis le début du XVIIIe siècle sur les activités d'extraction et de traitement des matières premières protohistoriques, avec ces caractères et ces structures spécifiques, l'exploitation du sel du $\mathrm{I}^{\mathrm{er}}$ millénaire avant notre ère comporte à l'évidence, à certains moments de son histoire, plusieurs éléments proprement « industriels ».

\subsection{Valeur d'usage et valeur d'échange}

Les discours des économistes sur la valeur d'usage et la valeur d'échange sont aussi vieux que la théorie économique elle-même puisqu'on en retrouve les prémices dans les travaux d'Adam Smith ou de David Ricardo ${ }^{45}$. On considère ainsi que toute marchandise, ici le sel, a une valeur d'usage qui tient à sa consommation dans le système économique concerné ; la valeur d'échange fait référence à une grandeur équivalente entre les différents termes de l'échange, qui peut être la monnaie métallique mais qui peut également se déporter sur d'autres marchandises si la monnaie n'est pas présente. Marx ${ }^{46}$ postule que toute marchandise possède ce caractère «bifide » d'être à la fois valeur d'usage et valeur d'échange. Dans le cas du sel, l'étude ethnologique de M. Godelier et les critiques des économistes qui s'en sont suivies sur la notion de «monnaie » des barres de sel baryua (Papouasie Nouvelle-Guinée) est particulièrement éclairantes ${ }^{47}$.

Il est certain que le sel a une valeur d'usage importante, en raison des multiples utilisations possibles qu'on lui connaît (cf. introduction). En ce qui concerne la valeur d'échange, il existe une littérature abondante sur la place du sel comme monnaie dans des sociétés traditionnelles et plusieurs hypothèses selon lesquelles le sel aurait pu jouer ce même rôle aux périodes protohistoriques $^{48}$. Il est vrai que la normalisation et le calibrage des pains de sels peut laisser

\footnotetext{
${ }^{42}$ KULL, 2003.

${ }^{43}$ STÖLlNER, 2002, p. 20-46.

${ }^{44}$ CNRTL, http://www.cnrtl.fr/definition/industrie (consulté le 28/02/2018)

45 JAUMOTTE, 2012.

${ }^{46}$ DuPUY, 2012, p.45-53.

${ }^{47}$ GODELIER, 1969

${ }^{48}$ MiLLOT-RICHARD, 2014, p.
} 
penser que ces marchandises ont pu servir de valeur étalon lors d'échange, de réserve de valeur; et c'est justement le fait de formater ce sel en lingots qui leur donne la valeur. De nombreuses marchandises prennent leur valeur d'échange parce qu'ils sont mis sous forme de lingots. C'est le cas du tabac, de l'or, d'autres éléments métalliques comme les lingots de bronze, et aussi du sel. Un exemple classique est le trafic caravanier du sel en Afrique saharienne et sub-saharienne aux périodes récentes ${ }^{49}$. Dans ces cas-là, on n'échange pas simplement du sel, mais bien des pains de sel, ce qui est tout à fait différent. Remarquons d'ailleurs que la valeur d'échange du sel augmente fortement avec son éloignement du site de production. Sans pouvoir qualifier les pains de sel protohistoriques de «monnaie», il convient de souligner qu'il s'agit de biens fortement calibrés, calibre variable selon le lieu de production et l'époque, qui lui confère une valeur d'échange spécifique, et probablement également une valeur d'usage à l'image des deux types de pains de sel produits simultanément dans les ateliers du Nord de la France et qui pourraient bien correspondre à deux qualités et types de sel différents, un sel gris âcre au goût et un sel blanc plus pur ${ }^{50}$.

\section{Premiers résultats et perspectives}

Un des apports de notre travail de recherche ${ }^{51}$, réside dans la comparaison et la confrontation avec l'exploitation du fer. Le choix de confronter ces deux matières premières se fonde en partie sur leur présence concomitante dans la zone d'étude. En effet, nous disposons de preuves archéologiques de l'extraction et du travail du minerai de fer dès le Hallstatt D3 et La Tène A pour la vallée de la Moselle, le nord de la Forêt-Noire, le Marckgräferland et le Jura souabe $^{52}$. Cette exploitation est supposée pour le reste de la Forêt-Noire mais également pour le massif vosgien où l'on manque de preuves archéologiques pour les périodes protohistoriques. Aussi, outre les deux massifs montagneux où l'exploitation du sel et du fer sont supposées, l'exploitation des deux matières premières ne s'effectue pas dans les mêmes régions. Les circulations qui en découlent sont-elles complémentaires, interdépendantes, autonomes ?

Nous ne reviendrons pas ici sur les modalités techniques de l'exploitation du fer ${ }^{53}$, mais le processus a en commun avec l'exploitation du sel de demander d'importantes quantités de bois et donnent lieu également à la fabrication de biens normalisés, ici les demi-produits ferreux $^{54}$. Il s'agit d'éléments en fer qui résultent du compactage des loupes de fer brutes de réduction qui sont ensuite mis en forme. Pour le Hallstatt on connaît uniquement des objets «bipyramidés » dont le poids, la forme précise et la qualité de métal varient, et, à partir de La Tène C, on voit l'apparition de demi-produits connus sous le nom de «currency bars ». Ces derniers sont davantage épurés et calibrés que les demi-produits bipyramidés. Le fait que les sociétés protohistoriques produisent des objets calibrés, pour le fer comme pour le sel, est significatif de la place qui leur est accordée dans les circuits d'échange.

Certaines des variables principales de l'étude d'un système économique sont le contrôle de la production et la consommation, à savoir la demande. Ce paramètre peut être approché en

\footnotetext{
${ }^{49}$ RITTER, 1981.

${ }^{50}$ WELLER, 2000

${ }^{51}$ Thèse en cours à l'Université Paris 1 (C. M-R)

${ }^{52}$ GASSMANN ET AL., 2005.

${ }^{53}$ BAUVAIS, 2007, p. 49-68.

${ }^{54}$ BERRANGER, 2014.
} 
archéologie par la relation entre le ou les sites de productions et la distribution, le type ou la densité des sites alentours. En se focalisant ici sur le Bade-Wurtemberg plusieurs pistes de réflexion peuvent être ouvertes.

Pour la période du Hallstatt (Fig.7), les sites dits «princiers » qui concentrent des biens de prestige, des matières premières (fer, corail, bronze, ambre etc.), et probablement un certain nombre d'artisans qualifiés, ne sont pas situés à proximité immédiate du seul site connu de production de sel. Toutefois des éléments de briquetage ont été retrouvés sur le site d'habitat de Hochdorf (Hallstatt D3-La Tène A) ${ }^{55}$ ce qui atteste une consommation de sel, mais en aucun cas un contrôle de la production principale.

L'étude et les cartes réalisées par Martin Hees (DFG-Projekt Viereckschanzen von Nordheim $)^{56}$ montrent par exemple que si des éléments de briquetage ont bien été retrouvés autour du site princier du Hohenasperg, le site reste éloigné des principales sources salées connues, et les concentrations de sites à briquetages sont situées clairement plus en aval de la vallée du Neckar. Il est donc là aussi difficile de faire un lien direct entre les sites princiers et l'exploitation du sel dans le Bade-Wurtemberg. Dans ce cas précis, le sel participait peut-être indirectement à créer la richesse des sites princiers. La production et la consommation du sel ne semblent pas en dépendre, et les circuits économiques du sel diffèrent de ceux des autres matières premières, comme le fer par exemple qui y semblent plus liế ${ }^{57}$.

En ce qui concerne la période laténienne (Fig. 8), on observe également des phénomènes intéressants. Les oppida laténiens, sites fortifiés qui jouent un rôle important dans la concentration et probablement la redistribution des biens dans les civitates gauloises $^{58}$, mais semblent ici en retrait des circuits de production et de consommation du sel. Ceux connus pour le Bade-Wurtemberg, comme Heidengraben bei Grabenstetten est localisé très loin de toutes les sources salées connues et seuls deux éléments de briquetage ont été retrouvés, dans une fosse, au niveau des remparts du site ${ }^{59}$.

En revanche, on remarque l'importance des Viereckschanzen notamment le long de la vallée du Neckar, à proximité de certaines sources salées et des concentrations de briquetage. On pourrait éventuellement supposer que ces sites « ruraux » jouent un rôle dans l'exploitation, la consommation, voire plus particulièrement la transformation du sel. En effet, sur le Viereckschanz de Nordheim (La Tène C2-D2), en plus de mille cinq cents fragments de briquetages $^{60}$ retrouvés $^{61}$, on note un nombre important d'ossements de suidés, notamment de membres antérieurs. On pense évidemment à la fabrication de salaisons et plus précisément de charcuterie. L'association au sein du même fossé détritique de ces deux mobiliers semble fortement faire pencher l'interprétation dans cette direction. Il faut sans doute là aussi complexifier nos modèles pour inclure ces sites d'utilisation et transformation du sel dans notre compréhension des circuits de production et de consommation. Plusieurs cas, nous y

\footnotetext{
${ }^{55}$ KLEIN, 1985, p.236.

${ }^{56}$ Nous souhaitons ici chaleureusement remercier Martin Hees pour son aide précieuse en termes d'accès à la donnée et à ses cartes de distribution. HEES, 2012, p. 377-390.

${ }^{57}$ MILLOT-RICHARD, 2014.

${ }^{58}$ FICHTL, 2000.

${ }^{59}$ WIELAND, 1996, p. 41 ; p. 257.

${ }^{60}$ KLEIN, 1985, p. 332 ; HEES, ou en préparation, DFG-Projekt Viereckschanzen von Nordheim.

${ }^{61}$ Soit le plus grand nombre de fragments enregistrés toutes périodes confondues, hors sites de production.
} 
invitent dans ces contextes protohistoriques : en Autriche, les quantités importantes de salaisons réalisées dès la fin de l'âge du Bronze directement sur la mine de Hallstatt ${ }^{62}$, celles probables sur le site de la Seille ${ }^{63}$, ou encore, éloignés des sites de production du sel, l'existence dans l'Aisne d'un enclos orienté vers la conservation alimentaire au sel marin sur l'oppidum de Villeneuve-Saint-Germain ${ }^{64}$ ou encore l'utilisation de sel dans le traitement métallurgique de métaux précieux sur celui de Condé-sur-Suippe ${ }^{65}$, voir la multiplication des utilisations du sel à la Tène finale dans le Pas-de-Calais sur le site des Brebières ${ }^{66}$.

\section{Conclusion}

La situation particulière des ressources salifères en contexte continental, rares et très ponctuelles, crée une rareté qui peut stimuler ou tendre la demande pour ce produit particulier. De ce point de vue, comme cela a pu être démontré autour des sources salées franccomtoises $^{67}$, la variable démographique et l'intensité de la demande qui en découle sont fondamentales. L'extraction et la transformation de la saumure en pain de sel a constitué à certains moments du Ier millénaire avant notre ère une « industrie » capable de mobiliser une main-d'œuvre nombreuse, comme c'est le cas dans la vallée de la Seille. Capable également de générer des produits fortement normalisés et très probablement des surplus, excédant largement les besoins locaux.

Sur la base de ces éléments archéologiques, nous devons à présent amorcer une réflexion sur la place des structures économiques du sel dans les sociétés protohistoriques nord-alpines. Le rapport avec les sites de «haut-rang », oppida ou sites princiers est particulièrement significatif puisqu'il nous permet de questionner les modèles théoriques jusque-là utilisés. Outre les multiples utilisations qu'on connaît au sel, la place majeure de la conservation alimentaire et la circulation de pièces de viande salée durant la Tène C-D doit également être prise en compte.

Le sel est une matière première invisible aujourd'hui mais dont l'exploitation et la distribution sont fondamentales à bien des égards. Mieux appréhender ces systèmes économiques permettra sans doute une meilleure approche des systèmes économiques protohistoriques dans leur ensemble.

\section{Bibliographie}

AleXIANU M., Weller O. et BRIGAND R., 2008. « Usages et enjeux autour des sources salées de Moldavie précarpatique, Roumanie », dans Weller O., Dufraisse A. et Pétrequin P. (dir.), Sel, eau et forêt. D'hier à aujourd'hui. Actes du colloque international, Saline Royale d'Arcet-Senans, 3-5 octobre 2006. Besançon : Presses Universitaires de Franche-Comté, Cahiers de la MSH Ledoux 12 (coll. Homme et environnement, 1), p. 49-72.

\footnotetext{
${ }^{62}$ PUCHER et al. 2013

${ }^{63}$ JOUANIN 2015

${ }^{64}$ WeLLER ET RoBert 1995 ; WeLLER ET DESFOSSES 2002

${ }^{65}$ PION et al. 1997

${ }^{66}$ LAGNEAU et GUTIERREZ 2017

${ }^{67}$ PETREQUIN et WELLER 2007
} 
BaUVAIs S., 2007. Evolution de l'organisation Des Activités de Forge Dans Le Nord Du Bassin Parisien Au Second Age Du Fer. Etudes Pluridisciplinaires de La Chaîne Opératoire En Métallurgie Du Fer. Belfort-Montbéliard.

BEAUPRE J., 1901. Le Briquetage de la Seille et les fouilles faites en 1901 à l'occasion du Congrès d'Anthropologie de Metz. Bulletin de la Société d'Archéologie Lorraine, p.227-273. Berranger M., 2009. Le Fer, Entre Matière Première et Moyen d'échange, En France Du VIIe Au Ier Siècle Av. J.-C. : Approches Interdisciplinaires. Direction P. Brun, Paris 1 Panthéon Sorbonne.

BertauX, J.-P., 1981. L'archéologie du sel en Lorraine, in Le Sel et Son Histoire, Dir Carbourdin. : Université de Nancy II.

Buchsenschutz, O., 2015. L'Europe Celtique à l'Age Du Fer. VIIIe-Ier Siècle. O. Buchsenschitz (Dir). (Nouvelle Clio). Paris.

CARLE W., 1965. Zur Frage der vor- und frühgeschichtlichen Salinen in Baden-Württemberg. Oberrheinische geologische Abhandlungen, 14, p. 141-166.

DAIRE M.-Y., 2003. Le Sel Des Gaulois, Errance (Collection des Hespérides).

DUPUY, F., 2012. «Monnaies "primitives" : polysémie et plurifonctionnalité », Monnaie antique, monnaie moderne, monnaies d'ailleurs... Métissage et hybridation, Colloques de la maison René-Ginouvès, p. 45-53.

FichtL S., 2005. La Ville Celtique : Les 'Oppida' de 150 Av. J.-C. à 15 Ap. J.-C.Errance.

FLEISCHER F., et LANDOLT M., 2012. « Entzheim-Geispolsheim (Bas-Rhin, Frankreich)- Zur Entwicklung einer Siedlungslandschaft in der frühen Eisenzeit », Wege Und Transport. Beiträge Zur Sitzung Der AG Eisenzeit Während Der80. Verbandstandung Des West Und Süddeutschland Verbandes Für Altertumsforschung. Nürnberg : BEIER \& BERAN. ARCHÄOLOGISCHE FACHLITERATUR LANGENWEISSBACH, 193-207.

FRIES-KNOBLACH J., 2001. Geratschaften. Verfahren und Bedeutung der eisenzeitlichen Sahsiedem in Mittelund Nordwesteuropa. Leipzig : University of Leipzig, Leipziger Forschungen zur Ur- und Frühgeschichtlichen Archäologie, 2.

GASSMANN, G., HAUTMANN, A., HÜBNER C., 2005, Forschungen zur keltischen Eisenerzverhüttung in Südwestdeutschland, Stuttgart.

Godelier M., 1969. «La monnaie de sel des Baruya de Nouvelle-Guinée », L'Homme, XI (2), p. 5-37.

Gouletquer P., Kleinmann D. et Weller O., 1994. «Sels et techniques », dans Daire M.Y. (dir.), Le sel gaulois. Dossiers de Centre de Recherche Archéologique d'Alet, Suppl. Q, p. 123-161.

HANSEN L., 2013. Bad Nauheim und das weisse Gold der Kelten. Neue Forschungen zur eisenzeitlichen Saline von Bad Nauheim, Wetteraukreis. Hessen Archäologie, p.91-93.

HeEs M., 2002. Production et Commerce du sel à l'Age du Fer en Baden-Württtemberg (Allemagne), in Weller O. (dir.), Archéologie Du Sel, Techniques et Société Dans La Pré et Protohistoire Européenne, Actes Du Colloque 12.2 Du XIVe Congrès de UISPP, Liège 2001, et de La Table Ronde Du Comité Des Salines de France, Paris 1998. Internationale Archäologie, ASTK, 3, Rahden, Verlag Marie Leidorf GmbH, 209-217. 
HeEs M., 2002. Siedlungsarchäologie Der Hallstatt Und Frülatènezeit Im Raum Heilbronn, Tübingen.

HeEs M., 2012. Die Bedeutung der vorgeschichtlichen Salzgewinnung in Siidwestdeutschland, Dans : V Nikolov \& K. Bacvarov (eds). Salt and Gold: The Role of Salt in Prehistoric Europe. Provadia \& Veliko Tarnovo, 2012, p. 277-286.

HeEs M., et SCHARF W, 2003. Transportbriquetage der Späthallstatt-/Frühlatènezeit aus dem Uffenheimer Gau, Beiträge zur Archäologie in Mittelfranken (7), 133-38.

HoMmEL W., 1940. Keltische und mittlealterliche Salgewinnung in Swäbisch Hall., Württembergisch Franken Neue Folge (20/21), 129-44.

Jaumotte C., 2012. Les Mécanismes de l'économie. De Boeck (Ouvertues économiques).

JOUANIN G., 2015. «Alimentation carnée et productions de salaisons chez les bouilleurs de sel hallstattiens de Marsal « La Digue » (Moselle) », Dans Omer F. et Roure R., Les Gaulois au fil de l'eau, Actes du XXXVIIe colloque AFEAF, Montpelier 2013, Bordeaux : Ausonius, Mémoires 39/2, p. 1055-1060

JUSSERET S., OLIVIER L., WATTEAU M., RIDDIFORD N., BRANCH N., Le Briquetage de la Seille (Moselle) : géoarchéologie et archéogréographie d'un complexe d'exploitation intensive du sel à l'âge du Fer, Les Gaulois au fil de l'eau, Actes $d u 37^{e}$ colloque international de l'AFEAF, Montpellier, 2015, p. 515-538.

KeunE J-B., Das Briquetage in oberen Seillethal. Jahrbuch der Gesellschaft für Anthropologie, Ethnologie, und Urgeschichte, XXXII, 11-12, p.119-125.

KLEIN F., 1985. Siedlungfunde Der Ausgehenden Späthallstatt Und Frühen Latènezeit Aus Württemberg., Tübingen.

Kost E., 1940. Die keltoschesiedlung über dem Hallquell im Kocher Tal in Swäbisch Hall., Württembergisch Franken Neue Folge (20/21), 39-111.

Kull B., 2003. Sole \& Salz Schreiben Geschichte. 50 Landesarchäologie, 150 Jahre Archäologische Forschung in Bad NauheimPh. von Zabern. Mainz am Rhein.

LAFFITE J.D., 2006, 2007, 2009. Moyenvic (Moselle) 'Les Crôleurs'. Contournement Est RD 955. Rétablissement RD914. Metz.

LAFFITE J.D., 2002. « Le briquetage de la Seille à Moyenvic (Moselle, France) au lieu-dit Les Crôleurs » dans Weller O. (dir.) Archéologie du sel. Techniques et sociétés dans la Pré- et Protohistoire européenne, Actes du colloque international, XIVe congrès UISPP, Liège (Belgique), sept 2001 et de la table ronde du Comité des Salines de France, Paris, mai 1998, Internationale Archäologie, ASTK, 3, Rahden, Verlag Marie Leidorf GmbH, p. 197-207.

LAGNEAU A., et GuTiERreZ C., 2017. « La consommation de sel dans le nord de la Gaule du IIIe au Ier siècle av. n.-è., Apport de la caractérisation pétrographique à travers l'étude des moules à sel de Brebières « Les Béliers » (Pas-de-Calais) ». In. Hoët-van Cauwenberghe C., Masse A., Prilaux G., dir. Sel et société, tome 1, techniques, usages, langage, Presses Universitaires du Septentrion, 2017, p. 131-161.

LANDOLT M., (dir.) 2008. Entzheim-Geispolsheim, Bas Rhin, Lotissement d'activités du quadrant 4. Entzheim "In der Klamm" et Geispolsheim "Schwobenfeld". Rapport de fouille préventive. Strasbourg. 
LE ROYER DE LA SAUVAGERE F.-F., 1740. Recherches sur la nature et l'étendue de ce qui s'appelle communément Briquetage de Marsal avec un abrégé de l'histoire de cette ville, et une description de quelques antiquités qui se trouve à Tarquimpole. Paris.

Millot-RichaRd C., 2015. 'Entre les enfants d'Hérodote et les enfants d'Adam Smith.' Pour une approche économique des données archéologiques., Archimède (2), 194-204.

MOINIER B., et WELler O., Le sel dans l'Antiquité ou les cristaux d'Aphrodite, Paris, Les Belles Lettres, 2015.

OliviER L., 2000. Le «Briquetage de la Seille», Nouvelles recherches sur une exploitation proto-industrielle du sel à l'Age du Fer, Antiquités Nationales 32, 143-73.

OLIVIER L., 2010. Nouvelles recherches sur le site de sauniers de premier âge du Fer de Marsal 'la Digue’ (Moselle), Antiquités Nationales (41), 127-60.

OliviER L., 2012. The 'Briquetage de la Seille' (Moselle, France) : an Iron Age protoindistrial salt extraction center., in Technologieentwicklung Und-transfer in Der HallstattUnd Latènezeit. Hallstatt : BEIER \& BERAN. ARCHÄOLOGISCHE FACHLITERATUR LANGENWEISSBACH, 31-44.

PetRequin P., et Weller O., 2007. «XVe siècle av. J.-C. : la reprise de la croissance démographique dans le Jura », dans : C. Mordant, H. Richard et M. Magny (eds.), Environnements et cultures à l'Âge du Bronze en Europe occidentale. Actes du $129^{\mathrm{e}}$ congrès CTHS, 2004, Besançon, Paris, ed. CTHS (coll. Documents préhistoriques du CTHS, 21), p. 197-210.

Petrequin P., et Weller O., 2008. «L'exploitation préhistorique des sources salées dans le Jura français. Application et critiques d'un modèle prédictif », dans Weller O., Dufraisse A. et Pétrequin P. (dir.), Sel, eau et forêt. D'hier à aujourd'hui. Actes du colloque international, Saline Royale d'Arc-et-Senans, 3-5 octobre 2006. Besançon: Presses Universitaires de Franche-Comté, Cahiers de la MSH Ledoux 12 (coll. Homme et environnement, 1), p. 255279. Pion P., Pommepuy C., Auxiette G., Henon B. et Gransar F., 1997. «L'oppidum de Condé-sur-Suippe/Variscourt (Aisne) (fin IIe-début Ier siècle avant J.-C.) : approche préliminaire de l'organisation fonctionnelle d'un quartier artisanal », dans G. Auxiette, L. Hachem et B. Robert (dir.), Espaces physiques espaces sociaux dans l'analyse interne des sites du Néolithique à l'Âge du Fer. Actes de colloque, L'analyse spatiale des sites du Néolithique à l'Âge du Fer, $119^{\mathrm{e}}$ Congrès national des sociétés historiques et scientifiques, 1994, Amiens. Paris, Comité des travaux historiques et scientifiques, p. 275-309.

Pucher E., BArth FE., SeEmann R., Brandstätter F., 2013. «Bronzezeitliche Fleischverarbeitung im Salzbergtal bei Hallstatt», 80th ed. Vienna : Mitteilungen der Prähistorischen Kommission, Österreichische Akademie der Wissenschaften, Philosophischhistorische Klasse, 2013. p. 11-134.

RIDDIFORD N., BRANCH N., JUSSERET S., OLIVIER L., \& GREEN C., 2016. Investigating the human - envrironnement relationship of early intensive salt production : A case study from the Upper Seille Valley, Lorraine, northeast France, Journal of Archaeological Science : Reports (10), p. 390-402.

SAILE T., 2002. « Le commerce du sel dans l'Europe centrale préhistorique » dans Weller O. (dir.) Archéologie du sel. Techniques et sociétés dans la Pré- et Protohistoire européenne, Actes du colloque international, XIVe congrès UISPP, Liège (Belgique), sept 2001 et de la 
table ronde du Comité des Salines de France, Paris, mai 1998, Internationale Archäologie, ASTK, 3, Rahden, Verlag Marie Leidorf GmbH, p. 223-231.

STÖLLNER T., 2002. Der Dürrnberg, sein Salzwesen und das Inn Salzach Gebiet als Wirtschaftsraum., in Dürrnberg Und Manching. Wirtschaftarchäologie Im Ostkeltischen Raum. Hallein/ Bad Dürrnberg: Dr Rudlof Habelt GmbH, 77-95.

VEECK W., 1940. Eine keltische Solesiederei in Schwäbisch Hall. Württembergisch Franken Neue Folge, 20/21, p. 112-128.

WELLER O., 2000. «L'exploitation du sel marin dans le nord de la France durant le second âge du Fer », dans Marion S. \& G. Blancquaert G. (dir.), Les installations agricoles à l'âge du Fer en France septentrionale, Actes du IIe colloque Les établissements ruraux de l'âge du Fer en France septentrionale, ENS, Paris, 1997. Paris, Presses de l'ENS, Études d'histoire et d'archéologie, 6, p. 237-250.

WELLER O., 2006 «Exemples ethnographiques d'organisation du travail : les différentes exploitations de sel en Nouvelle-Guinée », Techniques \& Culture, 46-47, , p. 51-61.

WeLLER O., 2015. «First salt making in Europe : an overview from Neolithic times », Documenta Praehistorica, 42, p. 185-196. http://dx.doi.org/10.4312/dp.42.12

Weller O., et ROBERT B., 1995. «Le commerce du sel au La Tène Final : une problématique enfin relancée », Revue Archéologique de Picardie, 1/2, p. 87-96.

Weller O., et Desfosses Y., 2002. «Les ateliers sauniers de Sorrus (Pas-de-Calais) : un apport majeur aux techniques de production de sel et à leur évolution durant le second âge du Fer », Dans O. Weller (dir.), Archéologie du sel. Techniques et sociétés, Actes du colloque 12.2 XIVe Congrès de UISPP, Liège 2001, et de la Table Ronde du Comité des Salines de France, Paris 1998. Internationale Archäologie, ASTK, 3, Rahden, Verlag Marie Leidorf GmbH, p. 63-80. 